\title{
ESTIMATION OF PARTICLE SIZE DISTRIBUTION FROM VIS/NIR SCATTERING MEASUREMENTS
}

\author{
A. Postelmans ${ }^{1 *}$, B. Aernouts ${ }^{1}$, R. Watté ${ }^{1}$, J. Jordens ${ }^{2}$, T. Van Gerven ${ }^{2}$, W. Saeys ${ }^{1}$ \\ ${ }^{1}$ KU Leuven, Department of Biosystems, MeBioS, Kasteelpark Arenberg 30 bus 2456, 3001 Leuven, Belgium \\ ${ }^{2}$ KU Leuven, Department of Chemical Engineering, ProcESS, Celestijnenlaan $200 f$ bus 2424, 3001 \\ Leuven, Belgium
}

Corresponding author: annelies.postelmans@kuleuven.be

The size of colloidal particles is an important quality characteristic of emulsions and suspensions as it is related to the stability and the general perception of the product. To monitor this physical quality property during production or storage, a measurement technique is required which can quantify the particle size distribution (PSD) in a rapid and non-destructive way. As such colloidal particles typically have a different refractive index than the surrounding medium, they scatter light. Therefore, the possibility to estimate the PSD and volume fraction of scattering particles from measured Vis/NIR scattering coefficient profiles was investigated in this study. Both a shape dependent and a shape independent PSD estimation method were elaborated and applied to estimate the PSD of the fat globules in milk. The shape dependent method approximates the PSD by a probability density function (e.g. Weibull). In case of a correct choice of the probability density function, this method results in good and robust estimations. In case of the shape independent method, the PSD is approximated by a weighted combination of B-splines. This provides extra flexibility, but can result in oscillations and artefact peaks in the estimated PSD if the regularization in not sufficient.

KEYWORDS: particle size distribution, light scattering, Vis/NIR spectroscopy, emulsions

\section{INTRODUCTION}

The quality of colloid systems, such as suspensions and emulsions, is determined by the chemical composition of the medium and dispersed particles, as well as the physical characteristics of the particles. The particle size distribution (PSD) and volume fraction of particles (VF) have an important effect on the product properties such as viscosity ${ }^{1}$, perception of creaminess of food emulsions ${ }^{2}$, etc. Therefore, PSD monitoring during production and storage of these colloids could promote early detection of an altering product quality. Optical measurements such as Vis/NIR spectroscopy have already found their way to the food industry for (on-line) process monitoring on a variety of products ${ }^{3}$, but the number of studies aiming at PSD monitoring is still very limited.

For PSD estimation, the link between particle size and light scattering can be used instead of e.g. light absorption. In case of spherical particles, the relationship between bulk scattering properties (scattering coefficient $\mu \mathrm{s}$, anisotropy factor $\mathrm{g}$ ) and particle size is described by Mie theory. In case of a known PSD, the bulk optical properties and light propagation can be simulated, as was shown by Aernouts et al. for polydisperse systems ${ }^{4,5,6}$. However, in most cases the inverse problem is of interest, to derive the PSD from non-destructive optical measurements. In literature, inverse PSD estimations are often starting from simulated or measured optical density $^{7,8,9}$. It is an ill-posed problem that needs regularization to select the solution that corresponds to a physically possible PSD and is consistent with literature knowledge. Depending on the assumptions about PSD shape, estimation methods can be divided in shape dependent and shape independent methods. The former assume a parametrized distribution form, while the latter impose conditions to the smoothness of the PSD.

In this study, both a shape dependent and a shape independent estimation method are elaborated. Their performance in estimating the PSD and volume fraction of milk fat globules in raw and homogenized milk based on the measured Vis/NIR bulk scattering coefficient profile is evaluated. 


\section{MATERIALS AND METHODS}

\section{Milk samples, PSD measurements and bulk optical properties}

Two raw bulk milk samples were divided into subsamples and ultrasonically homogenized for respectively $0 \mathrm{~s}$, $120 \mathrm{~s}, 1200 \mathrm{~s}$ for sample $1^{5}$, and $0 \mathrm{~s}, 30 \mathrm{~s}, 60 \mathrm{~s}, 120 \mathrm{~s}, 240 \mathrm{~s}, 480 \mathrm{~s}, 960 \mathrm{~s}$ for sample 2. Reference particle size distribution measurements were made with the laser diffraction technique (Mastersizer 3000, Malvern, UK). The contribution of casein micelles to the measured PSD was identified as the first mode of the bimodal PSD of raw milk. To obtain the PSD of only milk fat, this PSD of casein was subtracted from both raw and homogenized samples $^{5}$. Before optical measurements, all samples were 3-fold diluted. A double integrating spheres (DIS) and unscattered transmission set-up was used to measure the total reflectance, total transmittance and unscattered transmittance in the wavelength range of 0.55-1.8 $\mu \mathrm{m}$. Bulk optical properties were calculated following the Adding-Doubling routine ${ }^{10}$. A more elaborate description of the sample treatment and measurement methods can be found in 5 .

\section{Shape dependent PSD estimation}

In the shape dependent PSD estimation method, the PSD was assumed to be well approximated by a 2-parameter probability density distribution (e.g. a gamma distribution, or Weibull distribution for raw milk) ${ }^{11}$. The distribution parameters and the volume fraction of scattering particles were estimated by minimizing the sum of squared relative errors between the measured $\mu$ s profile and the $\mu$ s profile simulated for the estimated PSD.

$$
\min \sum_{i=1}^{N_{\lambda}}\left(\frac{\mu_{s, i}-\widehat{\mu_{s, l}}}{\mu_{s, i}}\right)^{2}
$$

The upper and lower boundary of the volume fraction milk fat were respectively 0.5 and $4 \% \mathrm{v} / \mathrm{v}$, which covers a wide range of possible fat contents in 3 fold diluted samples, given the $98 \%$ confidence interval for milk fat content in undiluted milk is $2.82-6.21 \% \mathrm{v} / \mathrm{v}^{12}$. The PSD estimation was made on the scattering coefficient at the wavelengths $0.6-0.75 \mu \mathrm{m}$ with step size $0.01 \mu \mathrm{m}$. This subset of wavelengths in the Vis region was determined by trial and error.

\section{Shape independent PSD estimation}

In the shape independent PSD estimation method, the PSD was approximated by a weighted sum of B-splines. The B-spline basis consisted of 30 B-splines of 4th order, equidistantly spaced on a logarithmic scale over the range of possible particle radii of 0-10 $\mu \mathrm{m}$. By directly fitting the splines to the measured PSD, it was ensured that the number of splines was sufficiently high to capture the curvature of the PSD, but not too high as this would result in artificial oscillations in the solution ${ }^{7}$. The spline weights were determined to satisfy the following inverse equation ${ }^{8}$ :

$$
\mu_{s}(\lambda)=\int_{r_{\min }}^{r_{\max }} P S D(r) \cdot \sigma_{s}(r, \lambda) d r=\int_{r_{\min }}^{r_{\max }} \sum_{j=1}^{N B} w_{j} \cdot B_{j}(r) \cdot \sigma_{s}(r, \lambda) d r=\sum_{j=1}^{N B} w_{j} \cdot A_{j}(\lambda)
$$

This means that in case of error-free measurements, the scattering coefficient profile of the weighted sum of $B-$ splines should be equal to the measured scattering coefficient profile $\mu s(\lambda)$. After discretization, the resulting system of equations was solved by non-negative least squares with Tikhonov regularization ${ }^{13,14}$.

$$
\min \left\|A w-\mu_{s}\right\|^{2}+\gamma\|L w\|^{2}, \quad w \geq 0
$$

The non-negativity constraint on the spline weights avoids negative values in the final estimated PSD, as this would be physically impossible. The regularization term contains the regularization parameter $\mathrm{Y}$ and regularization matrix $L$. The matrix $L$ is a discrete representation of the second derivative and penalizes non-smoothness. The parameter $\mathrm{y}$ determines the strength of this penalty. Its value was determined by minimizing the $\mathrm{V}$-curve criterion $^{15}$.

$$
\min \|\hat{\tau}-\tau\|^{2} \cdot\|\widehat{w}\|^{2}, \quad 0.001 \leq \gamma \geq 10
$$


An estimate of the volume fraction of scattering particles was calculated from the spline weights. The PSD estimation was made on the scattering properties from $0.55-1.8 \mu \mathrm{m}$ wavelength with a step size of $0.01 \mu \mathrm{m}$.

\section{RESULTS AND DISCUSSION}

\section{Shape dependent PSD estimation}

In the shape dependent estimation, a gamma probability density function was assumed since it was able to make an acceptable fit to the PSD of both raw and homogenized samples. This monomodal function was a good fit for the raw samples and samples homogenized for more than $120 \mathrm{~s}$ (Figure 1b). The more bimodal shape of the samples homogenized for 30-120 s could not be fully captured. For example, the estimated PSD of the sample ' $30 \mathrm{~s}$ ' lacks the first mode, but estimates the second mode quite well. The first mode at small particle radii results from the increase in small globules due to homogenization of the fat fraction. It might also contain a residual effect of the casein peak in case of a suboptimal removal of casein from the total milk PSD. To improve the estimates of bimodal PSD's, a combination of two probability density functions could be tested, e.g. two gamma distributions.

If the scattering coefficient is considered at all wavelengths $(0.55-1.8 \mu \mathrm{m})$, only the PSD of the raw sample could be estimated acceptably. In all other cases, the estimated PSD was a poor fit to the measured PSD and the estimated volume fraction equalled the upper or lower boundary value. When using the scatter coefficient profile for a subset of 16 wavelengths (0.6-0.75 $\mu \mathrm{m})$, all PSD estimation improved and the estimated volume faction no longer equalled a boundary value. This indicates that considering more wavelengths in the inverse estimation does not necessarily result in better estimations, as mentioned by Riefler \& Wriedt ${ }^{7}$.

The estimated volume fraction of milk fat showed realistic values $(1.36-2.19 \% \mathrm{v} / \mathrm{v})$. In theory, the value should be equal for all samples since homogenization has no effect on the total fat content of a sample. However, it was observed that the estimated volume fraction increased with increasing degree of homogenization.
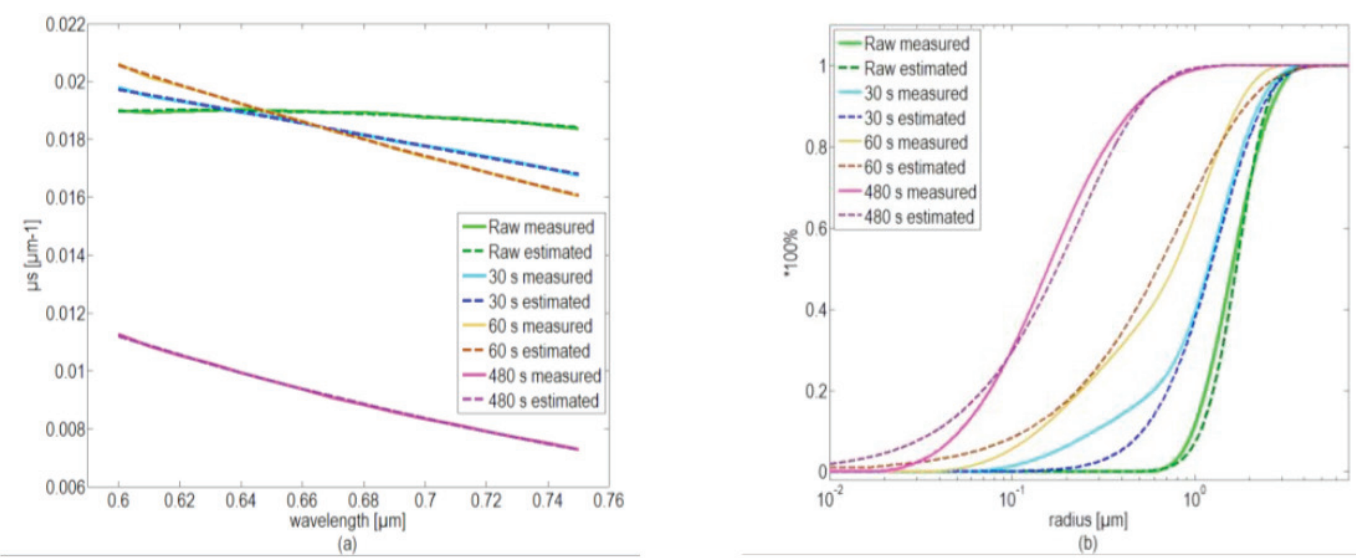

Figure 1. Measured (full lines) and estimated (dotted lines) scattering coefficient profile (a) and volume based particle size distribution (b) of raw and homogenized milk samples. Shape dependent estimation was made assuming a gamma distribution and the wavelength range of $0.6-0.75 \mu \mathrm{m}$.

\section{Shape independent PSD estimation}

In the shape independent method, the estimated PSD is a weighted combination of B-splines. The increased freedom in possible shapes compared to the shape dependent estimation should, in theory, be able to produce better fits. However, the extra flexibility might introduce artefacts e.g. oscillations or extra peaks ${ }^{14}$. This is illustrated in Figure 2b. For the raw milk sample, the position and width of the PSD are well-estimated, but an additional peak is introduced at large particle radii. For the ' $480 \mathrm{~s}$ ' sample, the PSD shape was well-estimated, except for a slight underestimation of the PSD width. For more bimodal PSD's, samples '30 s' and '60 s' in Figure 2 , the first mode was either ignored or underestimated, while a small extra mode at large radii appeared. It should be mentioned that in all cases the $\mu$ s profile of the estimated PSD gave an almost perfect fit to the measured $\mu \mathrm{s}$ profile (Figure 2a). 

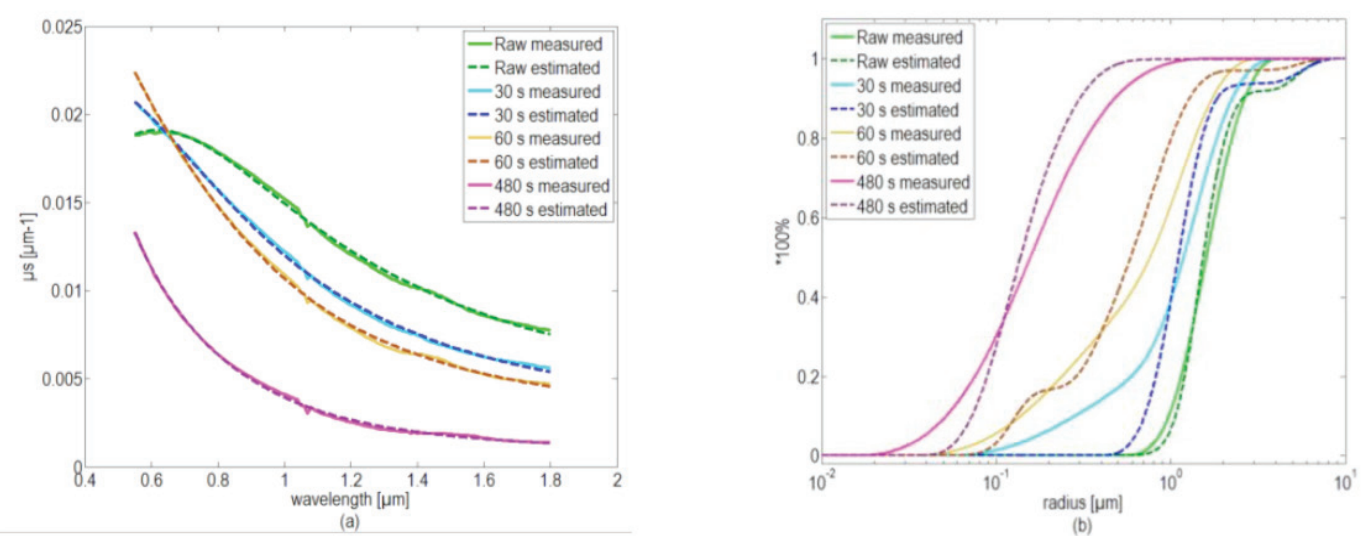

Figure 2. Measured (full lines) and estimated (dotted lines) scattering coefficient profile (a) and volume based particle size distribution (b) of raw and homogenized milk samples. Shape independent estimation was made using wavelengths of 0.55$1.8 \mu \mathrm{m}$.

Estimates for the volume fraction of fat varied from 1.45 to $3.46 \% \mathrm{v} / \mathrm{v}$ and increased with increasing degree of homogenization. This is possibly related to the tendency of the estimated PSD to underestimate the amount of small particles and overestimate the amount of large particles. As the particle volume, and thereby the volume fraction of fat, is proportional to the 3th power of the particle radius, the overestimation of large particles can significantly influence the estimated total amount of fat in the sample. Since light scattering by small particles increases with decreasing wavelength, inclusion of measurements in the UV-Vis wavelength region might increase the contribution of small particles to the $\mu$ s profile and thereby decrease the underestimation of their presence.

Differences between the estimated and measured PSD may be attributed to inaccuracy of the used refractive indices. Other possible sources are measurement errors in the optical and PSD measurements and changes in the sample in the time between those measurement. During this time, clusters may have formed which have influenced the scattering spectrum, while they are not present in the measured PSD. This might explain the extra peaks at large particle radii present in several estimated PSD's for the shape independent method. Finally, a suboptimal choice of the regularization parameter in the shape independent method may also have resulted in artefacts, while the gamma distribution used in the shape dependent method might be unable to perfectly fit the measured PSD.

\section{CONCLUSION}

Two methods have been elaborated for estimation of the PSD of colloidal samples from the bulk scattering coefficient profile: a shape dependent and a shape independent method. Both approaches have been applied to estimate the PSD and volume fraction fat of milk samples with a varying degree of homogenization. The bulk scattering coefficient profiles were calculated from double integrating sphere and unscattered transmission measurements with the inverse Adding-Doubling routine. The shape dependent PSD estimation was found to produce good results if the assumed probability density function provides a good approximation of the measured PSD. The shape independent estimation method on the other hand is more flexible with respect to the shape of the PSD, but was found to easily create artefacts. Therefore, it can be concluded that the shape independent method can provide a flexible tool for PSD estimation from Vis/NIR scattering profiles if the number of B-splines in the functional basis and the regularization are well-chosen. Estimated volume fractions of scatterers were in the correct range, but showed some homogenization dependency. The proposed inverse estimation forms an important step towards fast non-destructive optical methods for (on-line) determination of microphysical quality of emulsions and suspensions based on bulk optical properties. 


\section{Acknowledgments}

The authors gratefully acknowledge the Institute for the Promotion of Innovation through Science and Technology in Flanders for their financial support through the PhD grant of Annelies Postelmans (IWT-Flanders, grant 141687). Ben Aernouts is a postdoctoral fellow of the Research Foundation - Flanders (FWO, grant 12K3916N).

\section{References}

1. Servais, C.; Jones, R.; Roberts, I. Journal of Food Engineering, 2002, 51, 201-208.

2. Ahktar, M.; Stenzel, J.; Murray, B.; Dickinson, E. Food Hydrocolloids, 2005, 19, 521-526.

3. Huang, H.; Yu, H.; Xu, H.; Ying, Y. Journal of Food Engineering, 2008, 87, 303-313.

4. Watté, R.; Aernouts, B.; Saeys, W. Proc. of the 16th Int. Conf. on Near Infrared Spectroscopy, 2013, 15-22.

5. Aernouts, B.; Van Beers, R.; Watté, R.; Huybrechts, T.; Jordens, J.; Vermeulen, D.; Van Gerven, T.; Lammertyn,

J.; Saeys, W. Colloids and Surfaces B: Biointerfaces, 2015, 126, 510-519.

6. Aernouts, B.; Watté, R.; Van Beers, R.; Lammertyn, J.; Saeys, W.; Merchiers, M.; De Block, J. Optics Express, 2014, 22(17), 20223-20238.

7. Riefler, N.; Wriedt, T. Particle \& Particle systems Characterization, 2008, 25, 216-230.

8. Müller, D.; Wandinger, U.; Ansmann, A. Applied Optics, 1999, 38(12), 2346-2357.

9. Celis, M.-T.; Garcia-Rubio, L. Industrial \& Engineering Chemical Research, 2004, 43, 2067-2072.

10. Prahl, S. "Everything I Think You Should Know about Inverse Adding-Doubling," 2010, [Online]. Available: http://omlc.ogi.edu/software/iad/iad-3-9-10.zip.

11. Cabassi, G.; Profaizer, M.; Marinoni, L.; Rizzi, N.; Cattaneo, T. Journal of Near Infrared Spectroscopy, 2013, 21, 359-373.

12. Walstra, P.; Wouters, J.; Geurts, T. Dairy Science and Technology 2nd ed. (Taylor \& Francis Group), 2006, $763 \mathrm{p}$.

13. Wang, Y., Liang, G.; Pan, Z. Particuology, 2010, 8, 365-371.

14. Wang, L.; Sun, X.; Li, F. Applied Optics, 2012, 51(15), 2997-3005.

15. Liu, W.; Sun, X.; Shen, J. Optics \& Laser Technology, 2012, 44, 1-5. 